$N 71-26931$

NASA TECHNICAL

MEMORANDUM

NASA TM X-64582

ABSOLUTE STABILITY ANALYSIS OF ATTITUDE CONTROL

SYSTEMS FOR LARGE BOOSTERS

By S. M. Seltzer

Astrionics Laboratory

D. D. Siljak

University of Santa Clara

Santa Clara, California

April 2, 1971

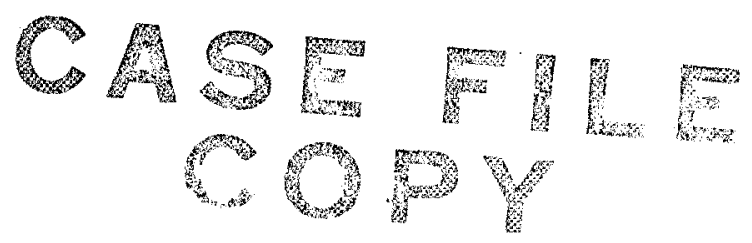

NASA

George C. Marshall Space Flight Center
Marsball Space Flight Center, Alabama 
TECHNICAL REPORT STANDARD TITLE PAGE

\begin{tabular}{|c|c|}
\hline $\begin{array}{l}\text { 1. REPORT NO. } \\
\text { NASA TM X-64582 }\end{array}$ & 3. RECIPIENT'S CATALOG NO. \\
\hline \multirow{2}{*}{$\begin{array}{l}\text { 4. TITLE AND SUBTITLE } \\
\text { Absolute Stability Analysis of Attitude Control } \\
\text { Systems for Large Boosters }\end{array}$} & $\begin{array}{l}\text { 5. REPORT DATE } \\
\text { April 2, } 1971 \\
\end{array}$ \\
\hline & 6. PERFORMING ORGANIZATION CODE \\
\hline $\begin{array}{l}\text { 7. AUTHOR (S) } \\
\text { S. M. Seltzer and D. D. Siljak }\end{array}$ & 8. PERFORMING ORGANIZATION REPORT \\
\hline \multirow{3}{*}{$\begin{array}{l}\text { 9. PERFORMING ORGANIZATION NAME AND ADDRESS } \\
\text { George C. Marshall Space Flight Center } \\
\text { Marshall Space Flight Center, Alabama } 35812\end{array}$} & 10. WORK UNIT NO. \\
\hline & 11. CONTRACT OR GRANT NO. \\
\hline & 13. TYPE OF REPORT \& PERIOD COVERED \\
\hline \multirow{3}{*}{$\begin{array}{l}\text { 12. SPONSORING AGENCY NAME AND ADDRESS } \\
\text { National Aeronautics and Space Administration } \\
\text { Washington, D. C. } 20546\end{array}$} & \\
\hline & Technical Memorandum \\
\hline & 14. SPONSORING AGENCY CODE \\
\hline
\end{tabular}

15. SUPPLEMENTARY NOTES

Prepared by Astrionics Laboratory, Science and Engineering

16. ABSTRACT

A method for performing an absolute stability analysis of attitude control systems for large launch vehicles is presented. Absolute stability of these systems is shown to be of a limited extent. The regions of absolute stability are computed by using a quadratic Liapunov function. The function is chosen to provide additional information about the exponential property of absolute stability. A system model is used to illustrate the method.

17. KEY WORDS

Booster

Attitude control

Stability analysis

Nonlinear stability theory
18. DISTRIBUTION STATEMENT

Unclassified - unlimited

19. SECURITY CLASSIF. (of this report)

Unclassified

20. SECURITY CLASSIF. (of this pate)

Unclassified

21. NO. OF PAGES

22. PRICE

22

$\$ 3.00$ 
TABLE OF CONTENTS

Page

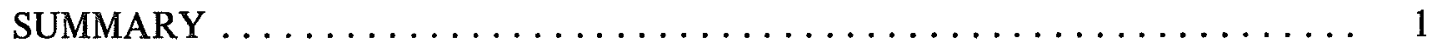

INTRODUCTION $\ldots \ldots \ldots \ldots \ldots \ldots \ldots \ldots \ldots \ldots \ldots \ldots \ldots \ldots \ldots$

ABSOLUTE STABILITY REGIONS $\ldots \ldots \ldots \ldots \ldots \ldots \ldots \ldots \ldots \ldots$

LARGE BOOSTER ATTITUDE CONTROL SYSTEM DESCRIPTION $\ldots \ldots \ldots .8$

APPLICATION $\ldots \ldots \ldots \ldots \ldots \ldots \ldots \ldots \ldots \ldots \ldots \ldots \ldots \ldots \ldots \ldots \ldots$

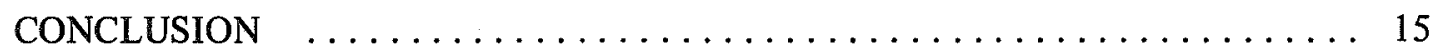

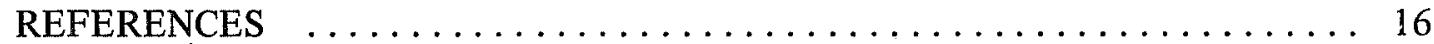




\section{LIST OF ILLUSTRATIONS}

Figure

Title

Page

1. Saturn attitude control system $\ldots \ldots \ldots \ldots \ldots$

2. Saturn navigation flow diagram $\ldots \ldots \ldots \ldots \ldots \ldots \ldots \ldots$

3. Simplified attitude control system $\ldots \ldots \ldots \ldots \ldots \ldots \ldots \ldots \ldots$

4. Typical attitude control system $\ldots \ldots \ldots \ldots \ldots \ldots \ldots \ldots \ldots \ldots$

5. Popov curve associated with equation (13) $\ldots \ldots \ldots \ldots \ldots \ldots$

6. Nonlinearity sector $\ldots \ldots \ldots \ldots \ldots \ldots \ldots \ldots \ldots \ldots \ldots$ 
TECHNICAL MEMORANDUM X-64582

\title{
ABSOLUTE STABILITY ANALYSIS OF ATTITUDE CONTROL SYSTEMS FOR LARGE BOOSTERS
}

\author{
SUMMARY
}

A method for performing an absolute stability analysis of attitude control systems for large launch vehicles is presented. Absolute stability of these systems is shown to be of a limited extent. The regions of absolute stability are computed by using a quadratic Liapunov function. The function is chosen to provide additional information about the exponential property of absolute stability. A system model is used to illustrate the method.

\section{INTRODUCTION}

The attitude control systems for large boosters can be analyzed in the framework of absolute stability. This method opens new avenues for applications of numerous strong results of absolute stability theory [1] to the control of large launch vehicles.

Since the linear part of the system is not asymptotically stable, the saturationtype characteristic of the hydraulic actuator used for rotating a gimbaled engine violates the sector condition, and the absolute stability of the attitude control system is shown to be of a limited extent. Regions of absolute stability are estimated by using quadratic Liapunov functions. The Popov frequency criterion and Yakubovich matrix inequalities are used in selecting an appropriate Liapunov function, which provides additional information about the exponential properties of absolute stability. Such properties guarantee a desired degree of rapidity of the transient process that takes place in the system if perturbations occur.

Significant advantages of the proposed method are: It is independent of the order of the system, and the algebraic operations involved in the computations are relatively simple and convenient for machine implementation. Moreover, the obtained results are valid not only for a particular nonlinearity but also for an entire class of nonlinear characteristics that satisfy certain general conditions.

Liapunov instability criteria and frequency domain techniques were used [2] for choosing the feedback control parameters in an attitude control system, which may result in stable operation. Conservative results were obtained so that insufficient 
information was provided for the estimation of parameters and states that assure system stability. In this direction, considerations of the attitude control systems for large launch vehicles by approximate methods [3] are more promising because they provide stronger "necessary conditions" in the estimation of stability regions in both the parameter and the state space. The approximate methods can be supplemented with the analysis of parametric absolute stability [1] to yield a useful addendum to the method presented herein.

\section{ABSOLUTE STABILITY REGIONS}

Attitude control systems for large boosters can be cast in the Lur'e-Postnikov class of nonlinear systems which are described by ${ }^{1}$

$$
\dot{x}(t)=\operatorname{Px}(t)+q \phi(\sigma), \sigma(t)=r^{T}(t)
$$

In equations $(1), \mathrm{x}(\mathrm{t})$ is an $\mathrm{n}$ vector - the state of the system; $\mathrm{P}$ is a constant $\mathrm{n} \times \mathrm{n}$ matrix; $\mathrm{q}$ and $\mathrm{r}$ are constant $\mathrm{n}$ vectors; and $\phi(\sigma)$ is a scalar, continuous, or discontinuous function with only isolated points of discontinuity of the first kind.

In the absolute stability analysis of the attitude control system, the following aspects are essential:

a. A necessary condition for absolute stability is that the linear part of the system in equations (1) described by the transfer function

$$
\chi(\lambda)=\mathrm{r}^{\mathrm{T}}(\mathrm{P}-\lambda \mathrm{I})^{-1} \mathrm{q}
$$

be stable; i.e., the matrix $P$ in equations (1) should be Hurwitz. However, the vehicle dynamics give rise to zero eigenvalues of $P$, and a transformation of equations (1) is necessary to achieve stability of the linear part.

b. The function $\phi(\sigma)$, which represents the nonlinearity of the system, is generally a saturation-type characteristic that is expected to belong to the class defined as

1. Capital Roman letters denote matrices, lower case Roman letters denote vectors, capital Greek letters denote sets, and lower case Greek letters denote scalars. The letter $\mathrm{t}$ is used only for time, and the letter $\mathrm{V}$ only for a Liapunov function. Vectors are considered as column matrices, and superscript $\mathrm{T}$ denotes the transpose. The notation $\mathrm{H}>0$ means that $\mathrm{H}$ is a positive definite matrix, and $\mathrm{I}$ is the identity matrix. 


$$
\begin{gathered}
\Phi_{\kappa}: 0 \leqslant \sigma \phi(\sigma) \leqslant \kappa \sigma^{2}, \kappa \leqslant \infty \\
\phi(0)=0
\end{gathered}
$$

Thus, the characteristic $\phi(\sigma)$ should be situated entirely inside the sector $[0, \kappa]$ of the $\sigma \phi$ plane. Since the matrix $\mathrm{P}$ is not Hurwitz, a saturation-type characteristic violates the sector condition; i.e., the inequality in equation (3) and the absolute stability property of equations (1) are of a limited extent. Therefore, the analysis is directed towards estimating the finite regions of absolute stability in the state space [4] .

c. In the application to the attitude control system, merely knowing that the system is stable is not sufficient. It is also important to provide information about the rapidity of the transient process that takes place in the system after possible perturbations. A common comparison function is the exponential function, and it will be shown that there exist two positive constants $(\rho, \delta)$ independent of the initial values $\mathrm{x}(0)=\mathrm{x}_{0}$, such that the system motion $\mathrm{x}(\mathrm{t})$ satisfies the inequality

$$
\|x(t)\| \leqslant \rho\left\|x_{0}\right\| e^{-\delta t}
$$

for all $\mathrm{x}_{0}, \mathrm{t} \geqslant 0$, and any $\phi(\sigma) \in \Phi_{\kappa}$. With this property, the system in equations (1) is said to be exponentially absolutely stable [1]. To achieve this kind of stability, the transformation mentioned in item a. must be aimed to make the matrix $\mathrm{P}+\delta \mathrm{I}$ Hurwitz. This, in turn, will limit the exponential property of absolute stability to a finite extent.

We begin the absolute stability determination of the system in equations (1) by adding to the right-hand side the zero identity $\kappa_{\delta}\left(\mathrm{qr}^{\mathrm{T}} \mathrm{X}-\mathrm{q} \sigma\right) \equiv 0$, so that equations (1) become

$$
\dot{x}(t)=\mathbb{P}_{t r} x(t)+q \phi_{t r}(\sigma), \sigma(t)=\mathrm{r}^{\mathrm{T}} \mathrm{x}(\mathrm{t})
$$

where

$$
\mathrm{P}_{\mathrm{tr}}=\mathrm{P}+\kappa_{\delta} \mathrm{qr}^{\mathrm{T}}, \phi_{\mathrm{tr}}(\sigma)=\phi(\sigma)-\kappa_{\delta} \sigma
$$

The constant $\kappa_{\delta}$ is chosen as the least number that makes the matrix

$$
\mathrm{P}_{\delta}=\mathrm{P}_{\mathrm{tr}}+\delta \mathrm{I}
$$


a Hurwitz matrix. This is a linear problem, and any of the linear methods (e.g., parameter method, root locus, etc.) can be used to select the minimum value for $\kappa_{\delta}$ so that all the poles of

$$
\chi_{\operatorname{tr}}(\lambda)=\frac{\chi(\lambda)}{1+\kappa_{\delta} \chi(\lambda)}
$$

are located inside the half-plane $\operatorname{Re} \lambda<-\delta \leqslant 0$.

Once the appropriate value of $\kappa_{\delta}$ is found, one applies the absolute analysis to equations (5). The exponential property of absolute stability of equations (5) is guaranteed by satisfying the Popov inequality

$$
\pi(\omega) \equiv \kappa^{-1}+\operatorname{Re} \chi_{\operatorname{tr}}(-\delta+j \omega)>0
$$

for all real $\omega \geqslant 0$.

As shown by Yakubovich, the condition in inequality (9) is necessary and sufficient for the existence of a Liapunov function

$$
\mathrm{V}(\mathrm{x})=\mathrm{x}^{\mathrm{T}} \mathrm{Hx}, \mathrm{H}=\mathrm{H}^{\mathrm{T}}
$$

having the derivative along solutions of equations (5) as

$$
-\dot{\mathrm{V}}=\left(\mathrm{x}^{\mathrm{T}} \mathrm{G}_{\delta} \mathrm{x}+2 \mathrm{x}^{\mathrm{T}} \mathrm{g \phi}+\kappa^{-1} \phi^{2}\right)+\left(\sigma-\kappa^{-1} \phi\right) \phi+2 \delta \mathrm{V},
$$

where

$$
-\mathrm{G}_{\delta}=\mathrm{HP}_{\delta}+\mathrm{P}_{\delta} \mathrm{T}_{\mathrm{H}},-\mathrm{g}=\mathrm{Hq}+\frac{1}{2} \mathrm{r}
$$

and $\mathrm{P}_{\delta}$ is given in equation (7). 
The matrix $\mathrm{H}>0$ satisfies the matrix inequalities

$$
\begin{aligned}
& \mathrm{G}_{\delta}-\kappa \mathrm{gg}^{\mathrm{T}}>0, \kappa \neq \infty \\
& \mathrm{G}_{\delta}>0, \mathrm{~g}=0, \kappa=\infty
\end{aligned}
$$

Since $\mathrm{V},-\dot{\mathrm{V}}-2 \delta \mathrm{V}>0$, we can always find a sufficiently small number $\epsilon>0$ such that

$$
-\dot{\mathrm{V}}-2 \delta \mathrm{V} \geqslant 2 \epsilon \mathrm{V}
$$

By separating the variables and integrating with respect to time, we obtain

$$
\mathrm{V}[\mathrm{x}(\mathrm{t})] \leqslant \mathrm{V}\left(\mathrm{x}_{0}\right) \mathrm{e}^{-2(\delta+\epsilon) t}, \mathrm{t} \geqslant 0
$$

Since

$$
\mu_{\mathrm{m}}\|\mathrm{x}\|^{2} \leqslant \mathrm{~V}(\mathrm{x}) \leqslant \mu_{\mathrm{M}}\|\mathrm{x}\|^{2}
$$

where $\mu_{\mathrm{m}}$ and $\mu_{\mathrm{M}}$ are the minimum and maximum eigenvalues of $\mathrm{H}$, from equation (15) follows the exponential property in inequality (4) of motion $x(t)$, where $\rho=\left(\mu_{\mathrm{M}} / \mu_{\mathrm{m}}\right)^{1 / 2}$.

To make the class $\Phi_{k}$ of nonlinear functions $\phi_{\operatorname{tr}}(\sigma)$ as large as possible, one applies the Popov graphical construction to inequality (9) and finds the maximum value of $\kappa$ for which that inequality is satisfied.

Let us now consider a saturation-type characteristic

$$
\phi(\sigma)= \begin{cases}\xi \sigma, & |\sigma| \leqslant \eta \\ \xi \eta \operatorname{sign} \sigma, & |\sigma|>\eta\end{cases}
$$


where $\xi, \eta$ are positive constants. Because of the transformation in equations (6), the characteristic $\phi_{\operatorname{tr}}(\sigma)$ violates the condition

$$
\sigma \phi_{\operatorname{tr}}(\sigma) \geqslant 0 \text { for }|\sigma|>\alpha, \alpha=\xi \eta \kappa_{\delta}^{-1}\left(1+\kappa_{\delta}^{2}\right)^{1 / 2}
$$

which is necessary for absolute stability of equations (5). Therefore, we enlarge the class of nonlinear functions and require that $\phi_{\operatorname{tr}}(\sigma)$ belongs to

$$
\begin{gathered}
\Phi_{\kappa, \alpha}: 0 \leqslant \\
\sigma \phi_{\operatorname{tr}}(\sigma) \leqslant \kappa \sigma^{2},|\sigma|<\alpha \\
\phi_{\operatorname{tr}}(0)=0
\end{gathered}
$$

Since $\mathrm{P}_{\delta}$ is Hurwitz and $\phi_{\operatorname{tr}}(\sigma) \in \Phi_{\kappa, \alpha}$, the origin $\mathrm{x}=0$ of the state space is locally exponentially stable. Therefore, a region $\Omega_{\delta}$ of exponential absolute stability is defined as the set of all points $x_{0}$ for which the motions $x(t)$ of equations (1) starting at $\mathrm{x}_{0}$ are exponentially absolutely stable; i.e., exponentially stable for any $\phi_{\operatorname{tr}}(\sigma) \epsilon \Phi_{\kappa, \alpha}$.

Once the maximum $\kappa$ is determined such that the Popov inequality (9) is satisfied, and $\phi_{\operatorname{tr}}(\sigma) \in \Phi_{\kappa, \alpha}$ for some $\alpha$, one is interested in finding the largest region $\Omega_{\delta}^{\mathrm{m}}\left(\Omega_{\delta}^{\mathrm{m}} \subseteq \Omega_{\delta}\right)$ defined by

$$
\Omega_{\delta}^{\mathrm{m}}=\{\mathrm{x}:|\sigma|<\alpha ; \mathrm{V}(\mathrm{x})<\beta\}
$$

where the number $\beta$ is determined from

$$
\beta=\min _{|\sigma(x)|=\alpha} V(x)
$$

Then, from equations (10) and (20), we obtain the conditions for $\beta$ as

$$
\begin{aligned}
& 2 \mathrm{Hx}+v \mathrm{r}=0 \\
& \mathrm{r}^{\mathrm{T}} \mathrm{x}+\alpha=0
\end{aligned}
$$


The solution of linear equations in equation (21) for $\mathrm{x}$ and the constant $v$ yields

$$
\beta=\alpha^{2}\left(r^{T} H^{-1} r\right)^{-1}
$$

The region of exponential stability is

$$
\Omega_{\delta}^{\mathrm{m}}=\left\{\mathrm{x}: \mathrm{x}^{\mathrm{T}} \mathrm{Hx}<\beta\right\}
$$

and the problem is reduced to finding the matrix $\mathrm{H}>0$.

As suggested in Reference 4, one can use the Kalman construction to determine a specific $\mathrm{H}$ from

$$
\mathrm{HP}_{\delta}+\mathrm{P}_{\delta} \mathrm{T}_{\mathrm{H}}=-\mathrm{uu}^{\mathrm{T}}
$$

if $\pi(\omega)$ in equality (9) is factored as

$$
\pi(\omega)=\frac{\theta(j \omega) \theta(-j \omega)}{\left|\mathbf{P}_{\delta}-\mathbf{j} \omega\right|\left|\mathbf{P}_{\delta}+j \omega I\right|}
$$

and the vector $u$ is chosen such that

$$
\mathrm{u}^{\mathrm{T}}\left(\mathrm{P}_{\delta}-\lambda \mathrm{I}\right)^{-1} \mathrm{q}=\frac{\theta(\lambda)}{\mid \mathrm{P}_{\delta}-\lambda \mathrm{II}}-\kappa^{-1 / 2}
$$

It is important to note that a different matrix $\mathrm{H}$ produces a different region $\Omega_{\delta}^{\mathrm{m}}$ with respect to extent and orientation, and it is desirable to select one that provides in some sense the best estimate $\widetilde{\Omega}_{\delta}^{\mathrm{m}}$ of the region of exponential absolute stability. Then, a set of $\mathrm{H}$ matrices can be generated directly from the matrix inequalities (13), which can be reduced to Sylvester inequalities involving the elements of $\mathrm{H}$. The best estimate $\widetilde{\Omega}_{\delta}^{\mathrm{m}}$ may be determined, for example, by minimizing the volume of $\Omega_{\delta}^{\mathrm{m}}$ over the set of generated $\mathrm{H}$ matrices, which is a simple matter for quadratic regions. 


\section{LARGE BOOSTER ATTITUDE CONTROL SYSTEM DESCRIPTION}

A typical attitude control system for a large booster is the Saturn launch vehicle (Fig. 1) [5]. The major components of such a system consist of a stabilized platform, a launch vehicle digital computer (LVDC), an analog control computer, rate gyroscopes, engine actuators, and, in some instances, body-fixed control accelerometers. The stabilized platform is a three-gimbal device whose inner gimbal is stabilized by three mutually orthogonal gas-bearing gyroscopes. Mounted on the inner gimbal are three pendulous integrating gyroscopic accelerometers. The platform performs two functions: its gimba1 angles provide vehicle spatial attitude, and its accelerometers detect inertial acceleration. The LVDC serves as the central point for all data flow and data processing; it solves the vehicle navigation and guidance equations, controls the sequence of flight events, and computes attitude error signals. As shown in detail in Figure 2, the attitude command signals are computed by comparing the actual attitude of the vehicle (as indicated by the gimbal angles of the stabilized platform) and the attitude angles required to guide the vehicle to its destination. The required attitude angles are calculated in the LVDC from the state of the vehicle as determined from stored information, stable platform accelerometer outputs, and onboard calculations indicated in Figure 2. The attitude error signals are converted into analog form and provided to the analog control computer that combines these signals with rate gy roscope signals and, in some cases (depending on the control law postulated), lateral accelerometer signals. From this information, it generates commands to the propulsion engine actuators and, when included, to the auxiliary propulsion system; these commands also point the thrust vector in the desired direction. For simplicity, it is assumed that the vehicle under consideration is rigid and flying above the sensible atmosphere. If rotational motion is considered in only the pitch plane, rotational rigid-body dynamics may be described by

$$
\ddot{\theta}+c \beta L=0
$$

where

$$
c \triangleq \frac{\ell\left(\partial R / \partial \beta_{L}\right)}{I}
$$

The term $\ell$ of equation (28) represents the distance between the engine gimbal point and the vehicle center of mass, $R$ represents the lateral component of engine thrust, $\beta_{L}$ is the angle in the pitch plane between the thrust vector and the longitudinal axis of the vehicle, and I represents the vehicle's moment of inertia about the pitch axis. The symbol $\theta$ represents the pitch attitude of the vehicle. 


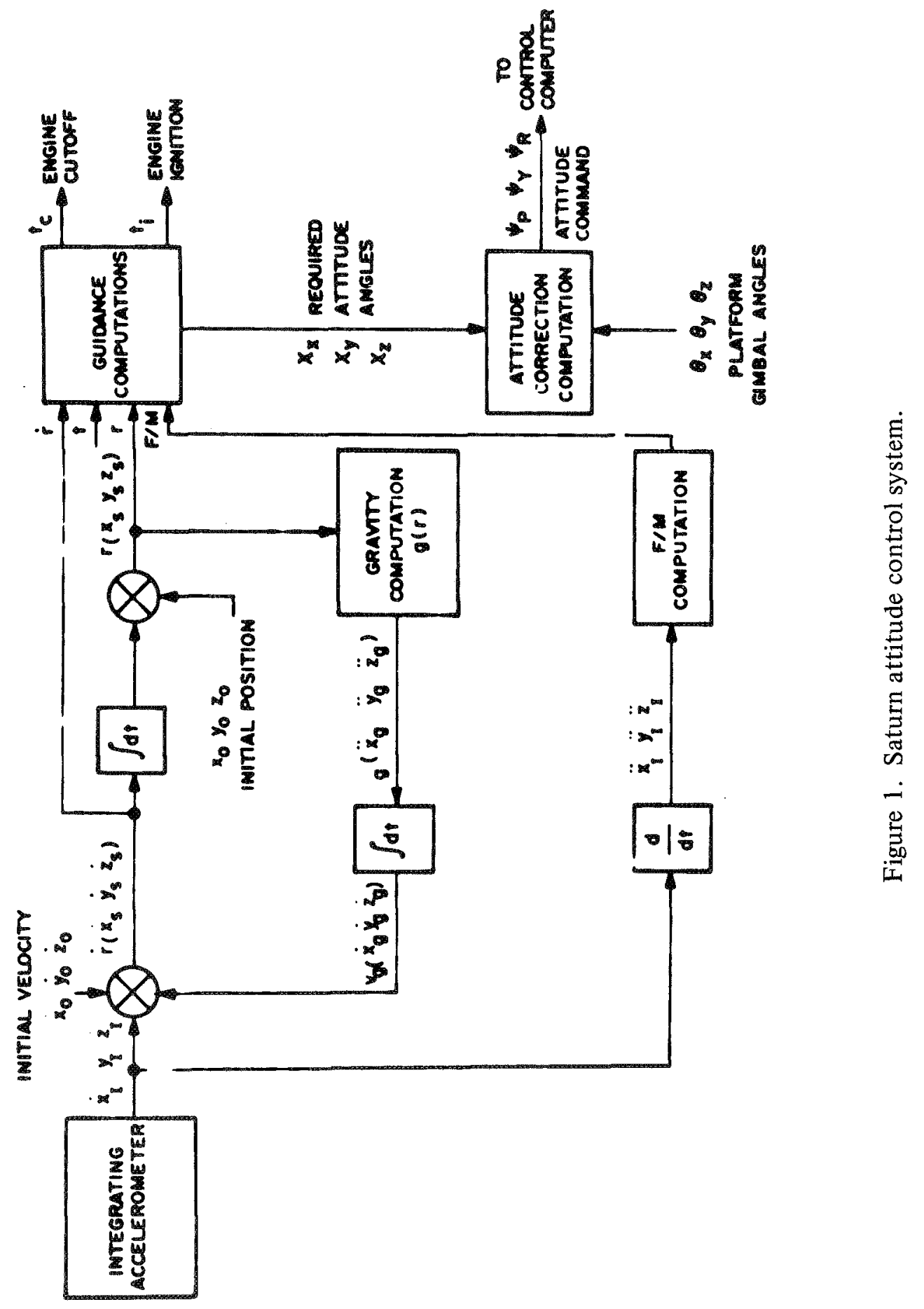




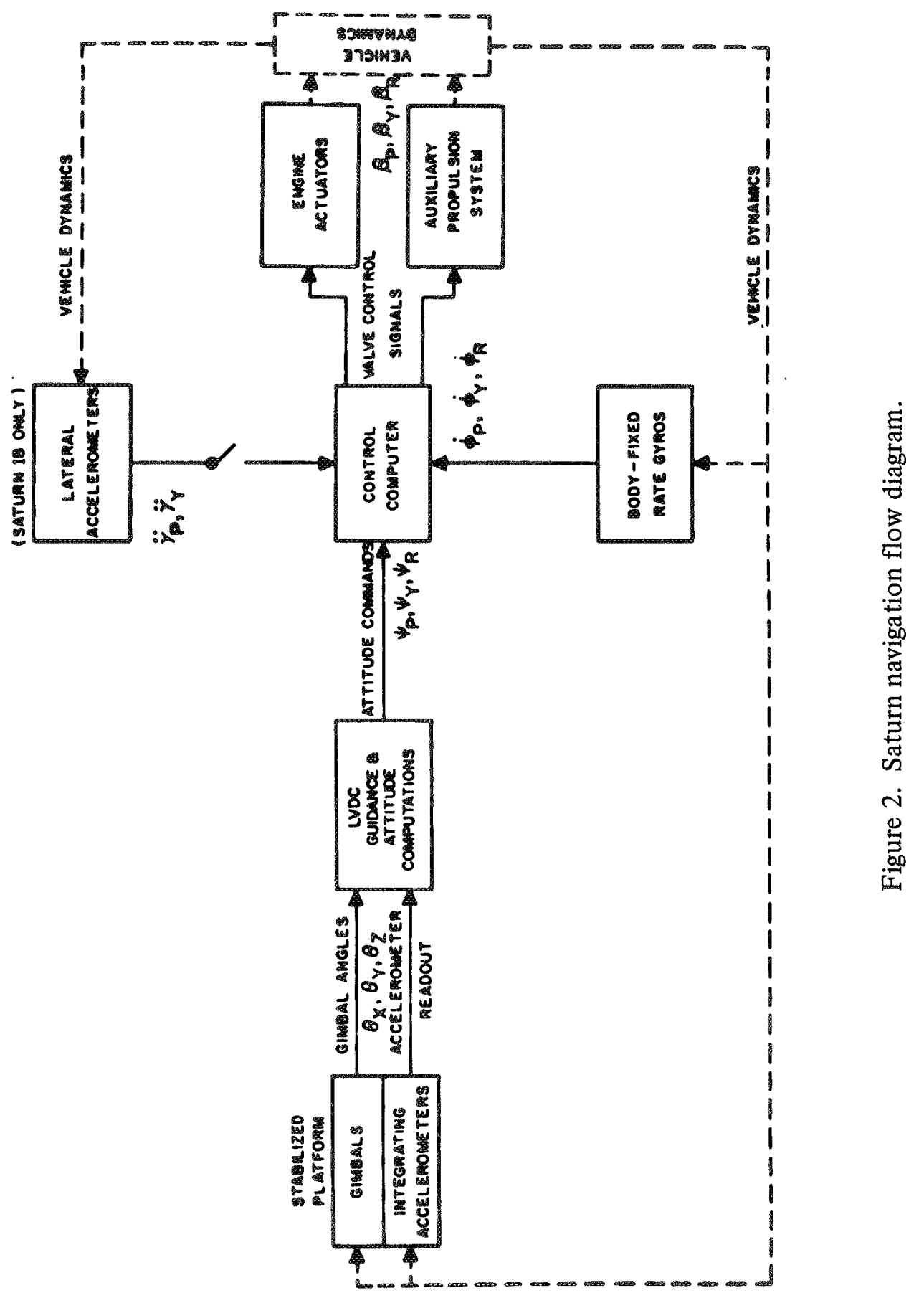


Numerous attitude control laws have been postulated for launch vehicles and used successfully. One postulate may be written as

$$
\sigma=\mathrm{a}_{0} \Psi+\mathrm{a}_{1} \dot{\theta}+\mathrm{a}_{2} \ddot{\theta}
$$

where $\sigma$ represents the commanded thrust vector angular rate; $\Psi$ is the measured attitude error; and $a_{0}, a_{1}$, and $a_{2}$ are adjustable control gains. If it is assumed that the engine actuator produces a thrust vector angular rate proportional to the commanded rate until a saturation limit $(\eta)$ is reached, the actuator may be modeled as equation (17), where $\phi(\sigma)$ represents $\dot{\beta_{\mathrm{L}}}$.

With the use of equations (27) and (29), the attitude control system (Fig. 1) may be simplified so that it appears as shown in Figure 3. For additional simplicity, it is assumed that the output of the lateral accelerometer is angular acceleration.

\section{APPLICATION}

Let us consider the simplified system model for the attitude control of a larger booster given in Figure 3. If a typical set of numerical values is chosen for $c, a_{0}, a_{1}$, and $\mathrm{a}_{2}$, such as 2,2,1, and 1.25, respectively, the figure may be further simplified (Fig. 4). This simple model, however, has all the characteristic properties of the considered class of systems necessary for a meaningful illustration of the outlined method of analysis. Since the method does not depend on the order of the system, it can be applied directly to more complex situations.

For equations (1), we take

$$
P=\left[\begin{array}{ccc}
0 & 1 & 0 \\
0 & 0 & 1 \\
0 & 0 & 0
\end{array}\right] ; \mathrm{q}=\left[\begin{array}{c}
0 \\
0 \\
-1
\end{array}\right] ; \mathrm{r}=\left[\begin{array}{c}
4 \\
2 \\
2.5
\end{array}\right]
$$

and

$$
\phi(\sigma)=\left\{\begin{array}{l}
10 \sigma, \quad|\sigma| \leqslant 0.1 \\
\operatorname{sign} \sigma,|\sigma|>0.1
\end{array}\right.
$$




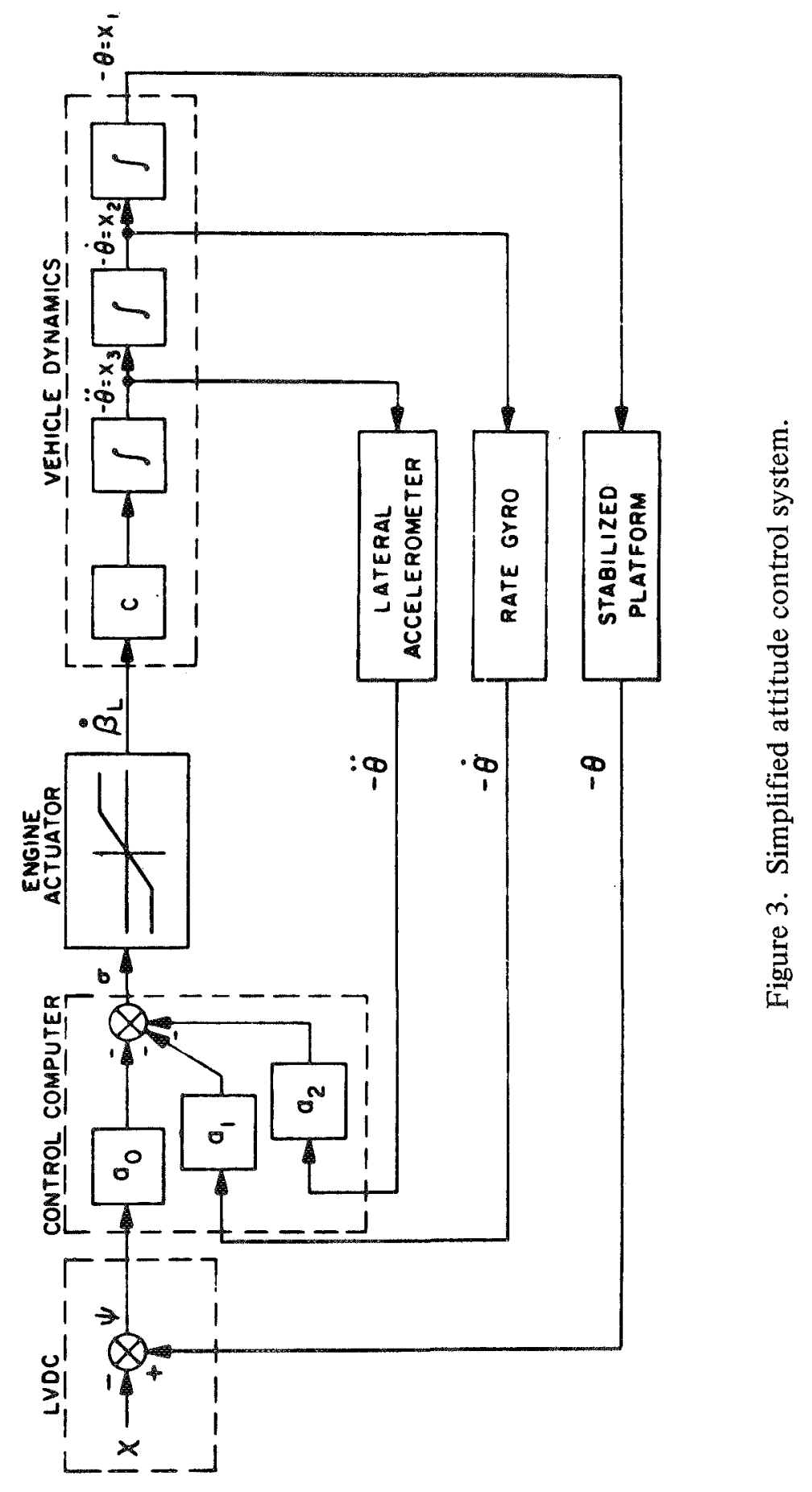


The transfer function in equation (2) of the linear part of the system is

$$
\chi(\lambda)=\frac{2.5 \lambda^{2}+2 \lambda+4}{\lambda^{3}}
$$

By applying a linear analysis, we can find that for ${ }^{\kappa}{ }_{0.2}=1.7$, all the poles of the transfer function in equation (8) computed as

$$
\chi_{\operatorname{tr}}(\lambda)=\frac{2.5 \lambda^{2}+2 \lambda+4}{\lambda^{3}+4.25 \lambda^{2}+3.4 \lambda+6.8}
$$

are located inside the half-plane $\operatorname{Re} \lambda<-0.2$.

According to the transformation in equation (6), the system to be analyzed is specified by

$$
\mathrm{P}_{\mathrm{tr}}=\left[\begin{array}{ccc}
0.2 & 1 & 0 \\
0 & 0.2 & 1 \\
-6.8 & -3.4 & -4.25
\end{array}\right] \quad \mathrm{q}=\left[\begin{array}{c}
0 \\
0 \\
-1
\end{array}\right] ; \mathrm{r}=\left[\begin{array}{l}
4 \\
2 \\
2.5
\end{array}\right]
$$

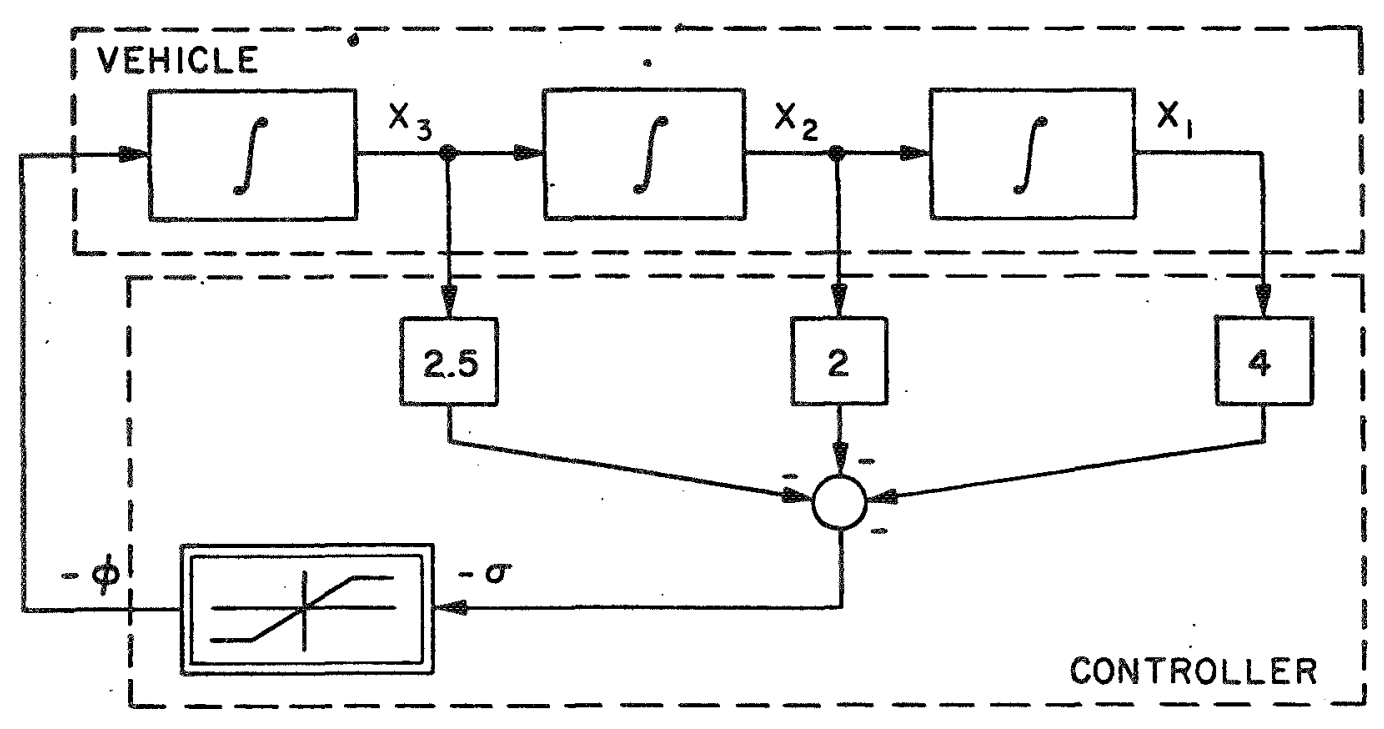

Figure 4. Typical attitude control system. 
Applying the Popov graphical test to inequality (9), the Popov diagram of Figure 5 is obtained for $\chi_{\operatorname{tr}}(\lambda)$ as in equation (33). As is clear from Figure 5, one can choose $\kappa=\infty$. Consequently, the system specified by equation (34) is stable exponentially in the entire sector $[0, \infty]$. Because of the transformation $\phi_{\operatorname{tr}}(\sigma)=\phi(\sigma)-1.7 \sigma$, the transformed saturation nonlinearity violates the sector $[0, \infty]$ at $|\sigma|=\alpha=11.6$ as shown in Figure 6 . Therefore, all functions $\phi_{\mathrm{tr}^{(}}(\sigma) \epsilon \Phi_{\infty}, 11.6$ that lie in the shaded region of Figure 6 are considered.

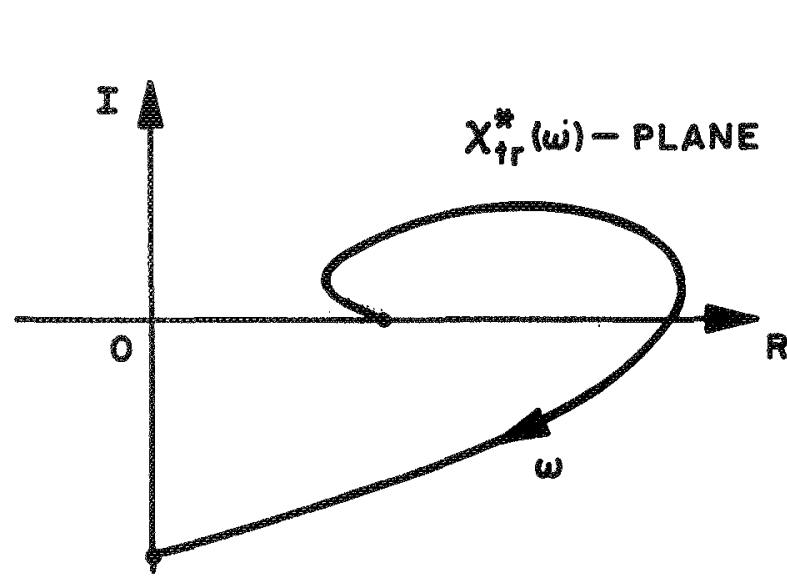

Figure 5. Popov curve associated with equation (33).

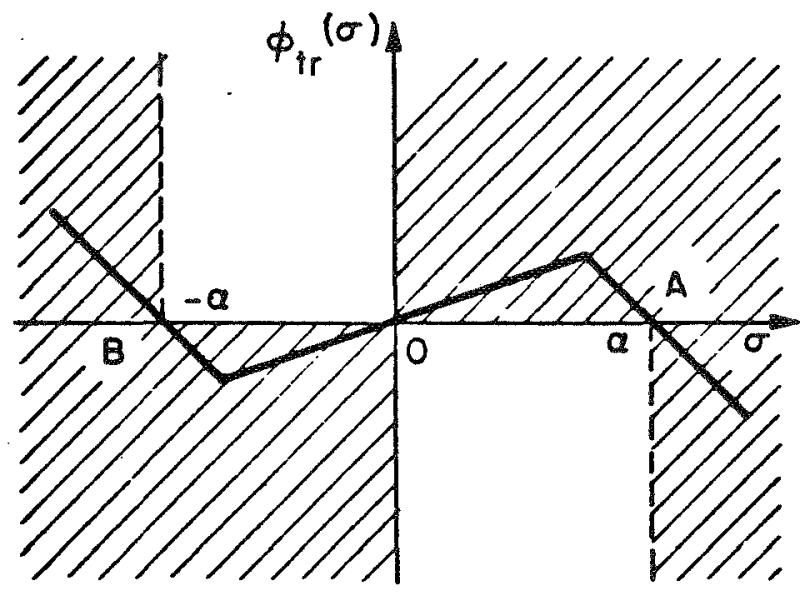

Figure 6. Nonlinearity sector.

Now to determine the region $\Omega_{\delta}^{\mathrm{m}}$ of exponential absolute stability for $\phi_{\mathrm{tr}}(\sigma) \epsilon \Phi_{\infty}, 11.6$ and $\chi_{\mathrm{tr}}(\lambda)$ given in equation (33), we take $\kappa=\infty$ and factor the corresponding expression $\pi(\omega)$ of inequality (9) as shown in equation (25). This operation yields the vector $u=[-0.22-0.74-2.85]^{\mathrm{T}}$ and the matrix

$$
H=\left[\begin{array}{ccc}
628.46 & 242.26 & 184.88 \\
242.26 & 444.02 & 974.51 \\
184.88 & 974.51 & 250.65
\end{array}\right]
$$

as specified by equations (24) and (26). Substituting this $H$ in equation (21), we obtain $\beta=0.44$ as defined in equation (22). Finally, we obtain an estimate of the region of exponential absolute stability for the system under investigation as

$$
\Omega_{0.2}^{\mathrm{m}}=\left\{\mathrm{x}: \mathrm{x}^{\mathrm{T}} \mathrm{Hx}<0.44\right\}
$$

where $H$ is the matrix specified in equation (35). 
It is of interest to note that the computed region $\Omega_{0.2}^{\mathrm{m}}$ in equation (36) corresponds to all $\phi_{\operatorname{tr}}(\sigma) \epsilon \Phi_{\infty}, 11.6$, which is the consequence of absolute stability. If we note that the transformed system is exponentially absolutely stable in the sector $[0, \infty]$, which implies that the original system has the same properties of stability for any nonlinearity that belongs to the sector $[1.7, \infty]$, we conclude that the relay characteristic $\phi(\sigma)=\operatorname{sign} \sigma$ can also be used in the system in equations (1) without affecting the region $\Omega_{0.2}^{\mathrm{m}}$ given in equation (36). The relay characteristic, being a discontinuous function of the first kind, may produce sliding motions. These kinds of absolute stability are shown in detail in Reference 1 , and no additional analysis is necessary after the region $\Omega_{\delta}^{\mathrm{m}}$ is computed.

The value of infinity was chosen for $\kappa$ to simplify the calculations in the example. However, it is reasonable to expect that better results in terms of a larger region of stability would accrue if a smaller value of $\kappa$ were chosen, as is clear from Figure 5 . In other words, a tradeoff probably exists between the size of the region of stability and the upper limit of the nonlinear sector. Of course, the upper limit cannot be less than the slope of the nonlinearity under consideration. In practice, a factor of safety would be included by insuring that $\kappa$ is selected so that the Popov sector always includes the nonlinearity under study, even under the influence of expected perturbations.

\section{CONCLUSION}

It has been shown how the regions of exponential absolute stability in the state space can be computed for attitude control systems for large boosters. A quadratic Liapunov function was used to estimate the regions. By considering the Lur'e-Postnikovtype Liapunov function "the quadratic form plus an integral of the nonlinearity," substantial improvements of the stability region estimation may be expected. This, however, would place stronger requirements either on the linear or nonlinear part of the system. Moreover, the numerical part of the analysis may be affected since the function is no longer quadratic.

It is of interest to note that the property of exponential stability makes it possible to consider perturbations on the system and determine a bound on the forcing function that guarantees that all the solutions remain bounded inside the computed region. This problem is solved in Reference 4.

Another aspect of the absolute stability analysis that is concerned with the choice of the vector $\mathbf{r}$ is not discussed herein. This problem, however, can be solved in the framework of parametric absolute stability as defined and developed in References 1 and 6. 


\section{REFERENCES}

1. Siljak, D. D.: Nonlinear Systems. John Wiley \& Sons, New York, 1969.

2. Pincura, S. C.: Application of Frequency Domain and Liapunov Instability Criteria to the Design of an Attitude Control System for a Large Booster. AIAA Guidance, Control, and Flight Mechanics Conference, Paper No. 69-853, Princeton, N. J., August 1969.

3. Seltzer, S. M.: Nonlinear Analysis of a Launch Vehicle Attitude Control System. Journal of Spacecraft and Rockets, Vol. 7, No. 3, March 1970, pp. 366-367.

4. Siljak, D. D., and Weissenberger, S.: Regions of Exponential Stability for the Problem of Lur'e. Regelungstechnik, Vol. 17, 1969, pp. $27-29$.

5. Decher, R., and Seltzer, S. M.: Saturn Launch Vehicles Astrionics System. Raumfahrtforschung, Vol. 1, January-March, 1968, pp. 33-37.

6. Siljak, D. D.: Singular Perturbation of Absolute Stability. Seminar on Structural Properties of Linear Systems, Politechnico di Milano, Milano, Italy, February 1971. 


\title{
APPROVAL
}

\section{ABSOLUTE STABILITY ANALYSIS OF ATTITUDE CONTROL SYSTEMS FOR LARGE BOOSTERS}

\author{
By S. M. Seltzer and D. D. Siljak
}

The information in this report has been reviewed for security classification. Review of any information concerning Department of Defense or Atomic Energy Commission programs has been made by the MSFC Security Classification Officer. This report, in its entirety, has been determined to be unclassified.

This document has also been reviewed and approved for technical accuracy.

Kam $\mathcal{C}$. Xosen Hain

HANS H. HOSENTHIEN

Chief, Research and Development

Analysis Office .

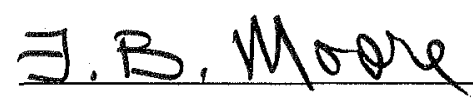

F. B. MOORE

Director, Astrionics Laboratory 


\section{DISTRIBUTION}

INTERNAL

DIR

DEP-T

PD-DIR

Dr. Murphy

PD-DO-DiR

Dr. Thomason

PD-DO-E

Mr. Schultz

S\&E-DIR

Mr. Weidner

Mr. Richard

S\&E-AERO-DIR

Dr. Geissler

Mr. Horn

S\&E-AERO-D

Dr. Lovingood

Dr. Worley

S\&E-AERO-G

Mr. Baker

S\&E-ASTR-DIR

Mr. Moore

Mr. Powell

S\&E-ASTR-A

Mr. Hosenthien

Dr. Borelli

Dr. Nurre

Dr. Clarke

Mr. Kennel

Mr. Jones

Mr. von Pragenau

Mr. Carroll

Mr. Daniel

Miss Flowers

Dr. Seltzer (25)
S\&E-ASTR-G

Mr. Mandel

Dr. Doane

Dr. Campbell

S\&E-ASTR-M

Mr. Boehm

S\&E-ASTR-S

Mr. Wojtalik

Mr. Brooks

Mr. Scofield

Mr. Chubb

Mr. Justice

Mr. Vallely

S\&E-CSE-DIR

Dr. Haeussermann

AD-S

A\&TS-MS-H

A\&TS-MS-IL (8)

A\&TS-MS-IP (2)

A\&TS-PAT

Mr. L. D. Wofford, Jr.

A\&TS-TU

Mr. Winslow (15)

PM-PR-M

EXTERNAL

University of Santa Clara

Electrical Engineering Department

Santa Clara, California 95053

Attn: Dr. Drago Siljak (25) 


\section{DISTRIBUTION (Concluded)}

EXTERNAL (Concluded)

Scientific and Technical Information Facility (2)

P. O. Box 33

College Park, Maryland 20740

Attn: NASA Representative (S-AK/RKT)

NASA

Washington, D. C. 20546

Attn: Mr. Carl Janow, REG

U. S. Army Missile Command

Redstone Arsenal, Alabama 35809

Attn: Mr. Jess Huff, RG

Mr. James McLean, RGN

Mr. Kelly Grider, RGN

NASA

Washington, D. C. 20546

Attn: Mr. Melvin F. Markey, MTG

Mr. Jerome Malament, MTG 